\title{
Case study of a sudden loss of vision in a diabetic patient: an unusual cause. Endogenous Klebsiella pneumoniae panophthalmitis and cerebellar abscess associated with pyogenic liver abscess
}

\author{
Rohit Sharma ${ }^{1}$, Sundus Sardar ${ }^{1}$, Waail Rozi ${ }^{1}$, Ibrahim Sa'id Khamees ${ }^{1}$, Ibrahim \\ Mohammad Obeidat ${ }^{1}$, and Wanis Hamad Ibrahim ${ }^{1}$ \\ ${ }^{1}$ Department of Internal Medicine, Hamad Medical Corporation
}

February 22, 2021

\begin{abstract}
Background: Community-acquired pyogenic liver abscesses due to Klebsiella pneumoniae is increasingly recognized across the world with more incidence in East Asian countries. Cases of endogenous endophthalmitis secondary to pyogenic liver abscesses with K1 serotype K. pneumoniae have been reported in the literature. We report a case of endogenous panophthalmitis along with cerebellar abscess occurring in a newly diagnosed diabetic patient secondary to pyogenic liver abscess. Case presentation: A middle-aged male of east Asian descent presented with sudden onset right eye vision loss associated with fever. Workup revealed newly diagnosed diabetes mellitus, panophthalmitis, cerebellar abscess, and pyogenic liver abscess due to K. pneumoniae. The patient, unfortunately, underwent right eye evisceration. Conclusion: Endogenous panophthalmitis due to K. pneumoniae is a fatal complication and can result in blindness. Multiple foci of infection can be present in such cases. Thorough workup, prompt antibiotics as well as surgical treatment may improve prognosis.
\end{abstract}

\section{Introduction}

K. Pneumoniae is an organism known to cause community-acquired Liver abscess syndromes. Such cases have been increasingly identified in the past decades particularly in patients with diabetes mellitus. Metastatic infectious complications due to this organism can occur. Endogenous endophthalmitis is a rare complication with panophthalmitis being more infrequent. It is very rare to encounter more than one infectious foci in such cases. We present a case of Pyogenic liver abscess with 2 metastatic foci; leading to panophthalmitis and cerebellar abscess.

This exemplifies the pertinence of thorough workup for infectious complications along with prompt medical as well as surgical treatment to prevent complications \& mortality. Despite treatment with parenteral and intravitreal antibiotics, visual outcomes are generally poor, savable vision is limited to eyes that receive early treatment.

\section{Case Presentation}

We present the case of a 56-year-old Indonesian man who presented to emergency department with fourday history of fever and two-day history of sudden-onset right-sided eye pain, redness, and loss of vision. The patient was in his usual state of health prior to the current presentation. Fever was associated with chills and headache, relieved with paracetamol. He experienced right eye redness, yellowish ocular discharge, painful right eye movement along with sudden onset loss of vision. He denied any history of trauma, ophthalmic conditions or surgeries, abdominal pain, nausea,vomiting, dysuria, cough, shortness of breath, 
or neck pain. Upon examination, he was found to be febrile (38.1 C), blood pressure 139/92 mmHg, heart rate of 116 beats per minute, respiratory rate of 22 breaths per min, and oxygen saturation of $99 \%$ on room air. Ophthalmological examination revealed right eyelid and periorbital swelling, impaired right visual acuity with increased intraocular pressure (Table 1). The rest of the physical examination was unremarkable. Right eye endophthalmitis was suspected and urgent computed tomography (CT) of both the orbits did not reveal any obvious radiodense foreign body in both orbital cavities.

Table 1 : Ophthalmological examination of both eyes

\begin{tabular}{lll}
\hline Examination & Right eye & Left eye \\
\hline Visual acuity & Hand movement & $6 / 6$ unaided \\
Intraocular pressure & $29 \mathrm{mmHg}$ & $14 \mathrm{mmHg}$ \\
Eyelids & Eyelid and periorbital swelling, minimal erythema & Normal \\
Lacrimal duct & Patent & Patent \\
Conjunctiva, sclera & Diffuse injection, chemosis and pus discharge & Clear \\
Cornea & Corneal edema, no epithelial defects & Clear \\
Anterior Chamber & 4 mm hypopyon & Quiet, deep \\
Iris & Hazy view to the upper part of iris & Normal \\
Pupil & Hazy view, irregular sluggish synechia & Pharmacologically dilated \\
Lense & Hazy view of the lens & Clear \\
Ocular Motility & Full, painless & Full, painless \\
Fundus & No view & Normal optic disc and macula, flat retina \\
\hline
\end{tabular}

Initial laboratory investigations revealed neutrophilic leukocytosis, hyponatremia and hyperkalemia (Table 2 ). Investigations were appreciative of an infectious process along with newly-diagnosed Type 2 diabetes mellitus and diabetic ketoacidosis (DKA). The patient was commenced on DKA protocol and empirically started on ceftriaxone plus vancomycin, which was later escalated to meropenem. He underwent right anterior chamber tap, vitreous tap and intracameral and intravitreal injection with vancomycin, ceftazidime, and voriconazole.

Table 2. Initial laboratory investigations

\begin{tabular}{lll}
\hline Investigation & Value & Reference range (male) \\
\hline WBC & $20.7 \times 10^{\wedge} 3 / \mu \mathrm{L}$ & $4-10 \times 10^{\wedge} 3 / \mu \mathrm{L}$ \\
Absolute Neutrophil Count & $17 \times 10^{\wedge} 3 / \mu \mathrm{L}$ & $2.0-7.0 \times 10^{\wedge} 3 / \mu \mathrm{L}$ \\
RBC & $5.2 \times 10^{\wedge} 6 / \mu \mathrm{L}$ & $4.5-5.5 \times 10^{\wedge} 6 / \mu \mathrm{L}$ \\
Hemoglobin & $15.6 \mathrm{~g} / \mathrm{dL}$ & $13-17 \mathrm{~g} / \mathrm{dL}$ \\
Platelets & $296 \times 10^{\wedge} 3 / \mu \mathrm{L}$ & $150-400 \times 10^{\wedge} 3 / \mu \mathrm{L}$ \\
Sodium & $130 \mathrm{mmol} / \mathrm{L}$ & $136-145 \mathrm{mmol} / \mathrm{L}$ \\
Potassium & $5.6 \mathrm{mmol} / \mathrm{L}$ & $3.5-5.1 \mathrm{mmol} / \mathrm{L}$ \\
Chloride & $93 \mathrm{mmol} / \mathrm{L}$ & $98-107 \mathrm{mmol} / \mathrm{L}$ \\
Bicarbonate & $20 \mathrm{mmol} / \mathrm{L}$ & $22-29 \mathrm{mmol} / \mathrm{L}$ \\
Glucose & $19.6 \mathrm{mmol} / \mathrm{L}$ & $3.3-5.5 \mathrm{mmol} / \mathrm{L}$ \\
Albumin ( blood) & $27 \mathrm{~g} / \mathrm{L}$ & $35-52 \mathrm{~g} / \mathrm{L}$ \\
Beta Hydroxy Butyrate & $2.69 \mathrm{mmol} / \mathrm{L}$ & $0.03-0.30 \mathrm{mmol} / 1$ \\
Venous pH & 7.28 & $7.31-7.41$ \\
Albumin corrected Anion gap $(\mathrm{AG})$ & $20.3 \mathrm{mmol} / \mathrm{L}$ & $3-11 \mathrm{mmol} / \mathrm{L}$ \\
Albumin corrected Delta gap & $8.3 \mathrm{mmol} / \mathrm{L}$ & $0 \mathrm{mmol} / \mathrm{L}$ \\
Albumin corrected Delta Ratio & 2.1 & A ratio $>2 \mathrm{reflects}$ high anion-gap acidosis and a concurrent metabc \\
Total bilirubin & $8 \mu \mathrm{mol} / \mathrm{L}-$ & $0-21 \mu \mathrm{mol} / \mathrm{L}$ \\
Alkaline phosphatase & $237 \mathrm{U} / \mathrm{L}$ & $40-129 \mathrm{U} / \mathrm{L}$ \\
& &
\end{tabular}




\begin{tabular}{lll}
\hline Investigation & Value & Reference range (male) \\
\hline ALT & $79 \mathrm{U} / \mathrm{L}$ & $0-41 \mathrm{U} / \mathrm{L}$ \\
AST & $35 \mathrm{U} / \mathrm{L}$ & $0-40 \mathrm{U} / \mathrm{L}$ \\
C reactive Protein & $187.2 \mathrm{mg} / \mathrm{L}$ & $0-5 \mathrm{mg} / \mathrm{L}$ \\
Procalcitonin & $29.10 \mathrm{ng} / \mathrm{mL}$ & $>2.0 \mathrm{ng} / \mathrm{mL}$ represents a high risk of sever sepsis and/or septic sho \\
Lactic acid & $2.2 \mathrm{mmol} / \mathrm{L}$ & $0.5-2.2 \mathrm{mmol} / \mathrm{L}$ \\
HbA1C & $13.2 \%$ & $<6 \%$ \\
\hline
\end{tabular}

A contrast-enhanced CT abdomen revealed a large lobulated and multiloculated intrahepatic lesion with fluid density content, occupying the right lobe involving segment VI and VII, measuring $8.1 \times 6.7 \times 7.4$ $\mathrm{cm}$ (in anteroposterior, transverse, and coronal dimensions), with irregular and thick peripheral as well as septal enhancement, surrounded by tissue edema and minimal free fluid and fat stranding in the subhepatic region. The finding was suggestive of hepatic abscess (Figure 1 ). He underwent ultrasound-guided drainage of the right liver lobe abscess and yellowish pus was aspirated. Cultures from the aqueous and vitreous fluid revealed Klebsiella pneumoniae. Pus culture from the liver abscess grew the Klebsiella pneumoniae with similar sensitivity. Two sets of blood cultures along with fungal cultures were negative.

Magnetic resonance imaging (MRI) head and orbit was done which showed extensive inflammatory changes involving the right orbit, preseptal, postseptal regions and vitreous chamber with diffusion restriction suggestive of right eye panophthalmitis (as the inflammatory changes extend beyond the scleral lining by definition). The right lens was subluxed laterally and the medial aspect of the uvea and sclera was thickened, irregular and detached laterally as along with the presence of a hemorrhagic component in the medial aspect of the right lobe (Figure2 and 3 ). These findings may be attributed to extensive inflammatory changes or could be related to the recent surgical intervention. A ring-enhancing lesion with central diffusion restriction was noted in the inferior aspect of the left middle cerebellar peduncle measuring $11 \times 7 \mathrm{~mm}$, representing abscess along with moderate perilesional vasogenic edema (Figure 4 ).

Based on the culture and sensitivity of the organism, antibiotics were shifted to ceftriaxone for better CNS penetration. The patient, unfortunately, had to undergo right eye evisceration for source control. He was discharged on oral trimethoprim-sulfamethoxazole with a follow-up MRI head to document the clearance of infection.

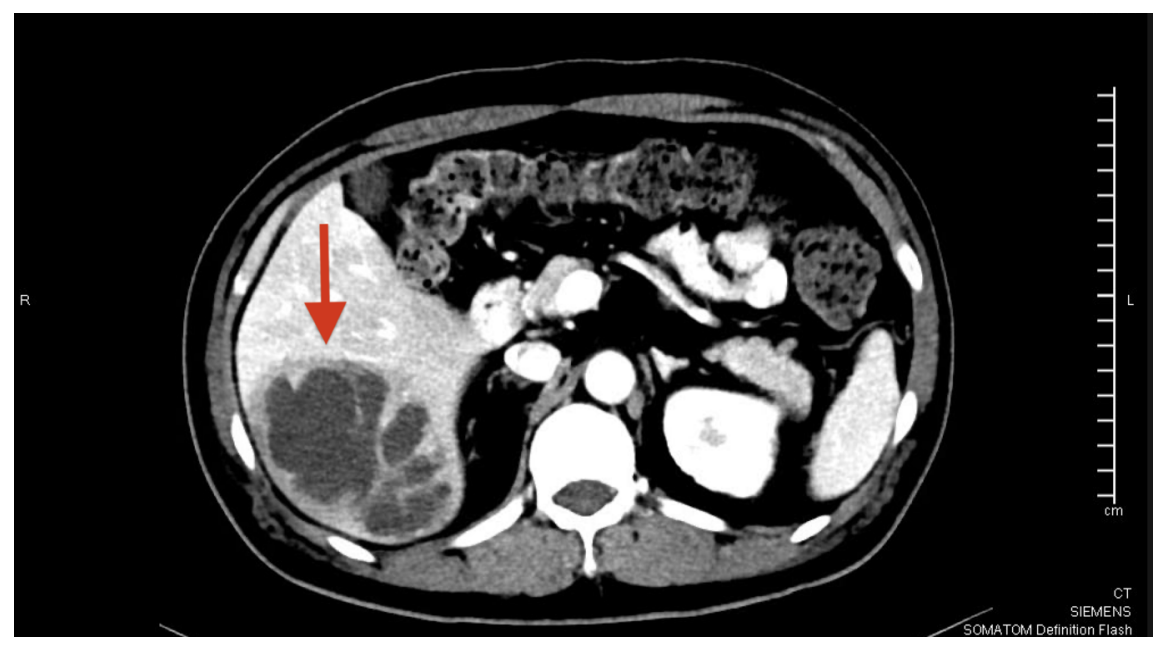

Figure 1: CT scan of the abdomen showing hepatic abscess (Red arrow) 


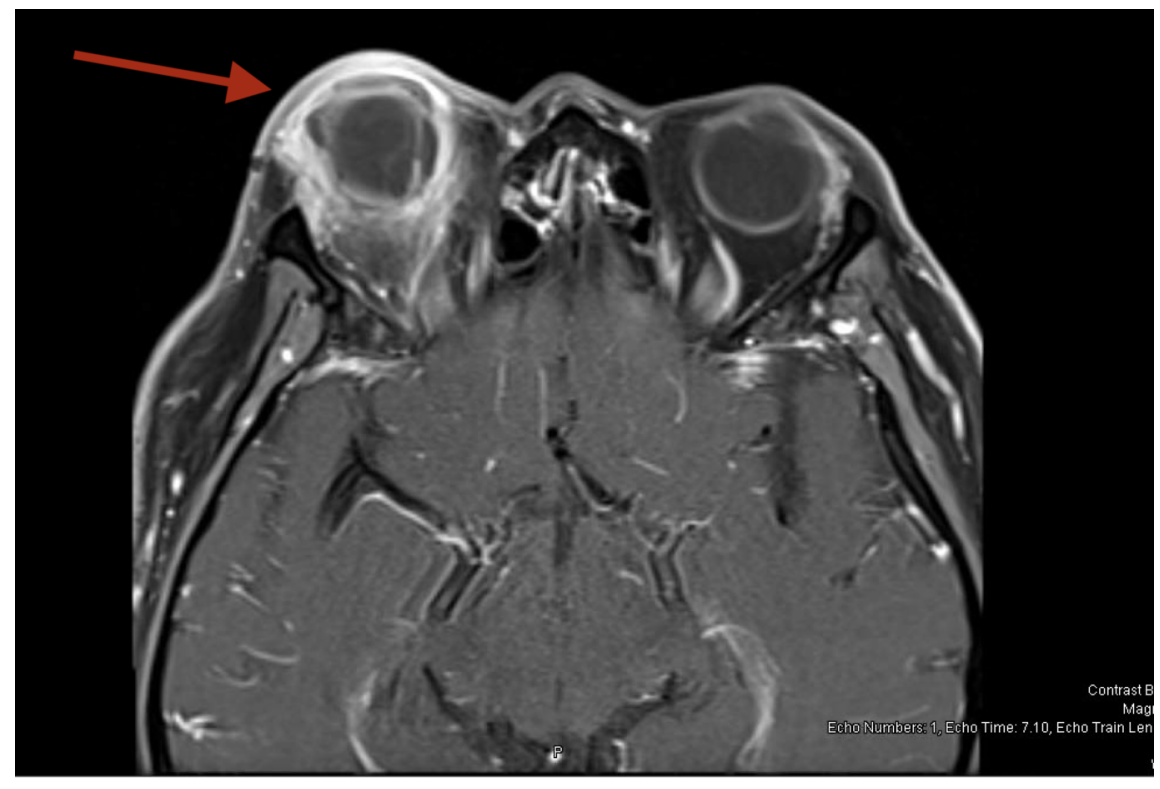

Figure 2: T1 weighted transverse post-contrast MRI orbit showing right pan-orbital enhancement.( marked by red arrow) 


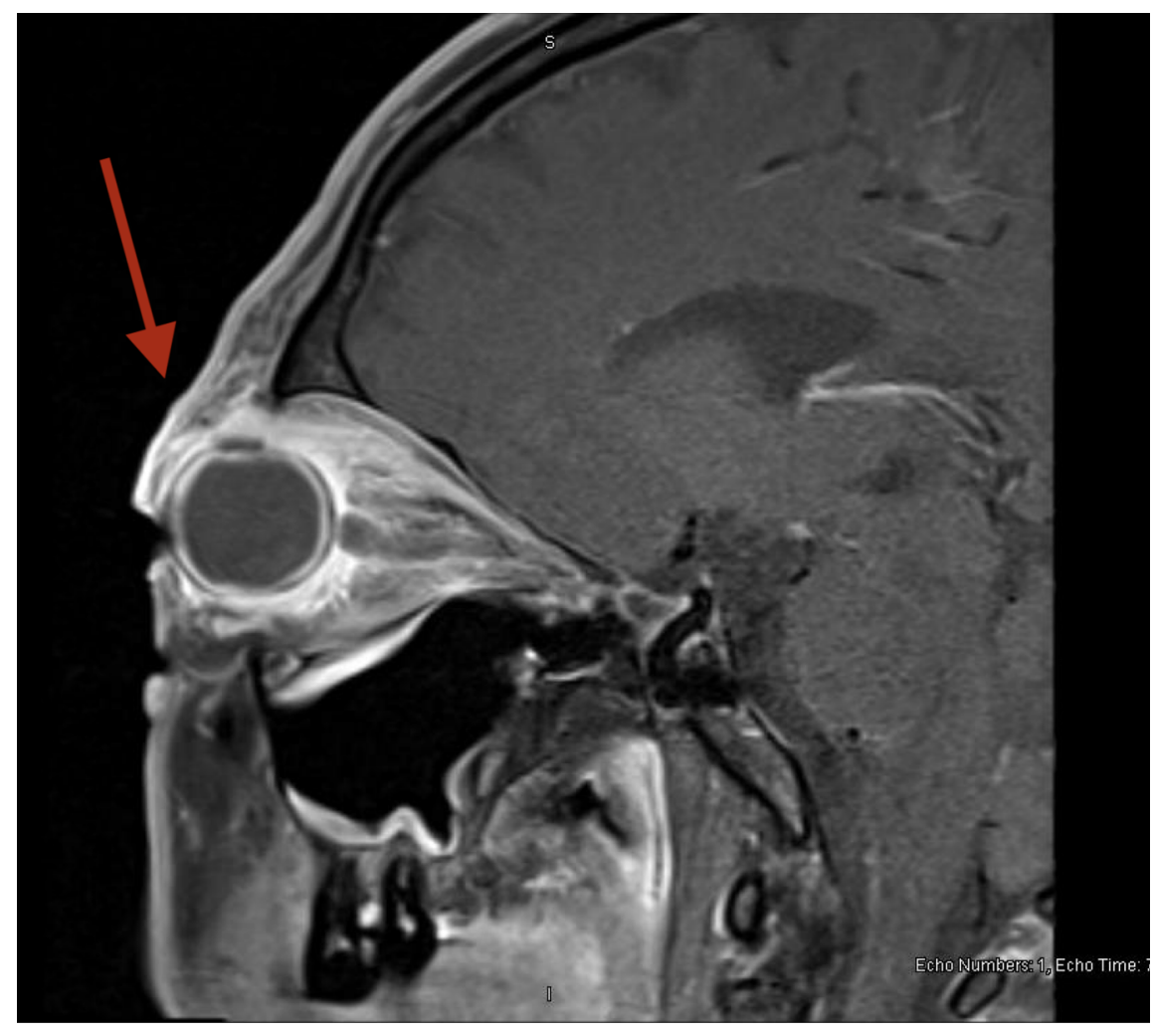

Figure 3: T1 weighted sagittal post-contrast MRI orbit showing right pan-orbital enhancement (marked by red arrow). 


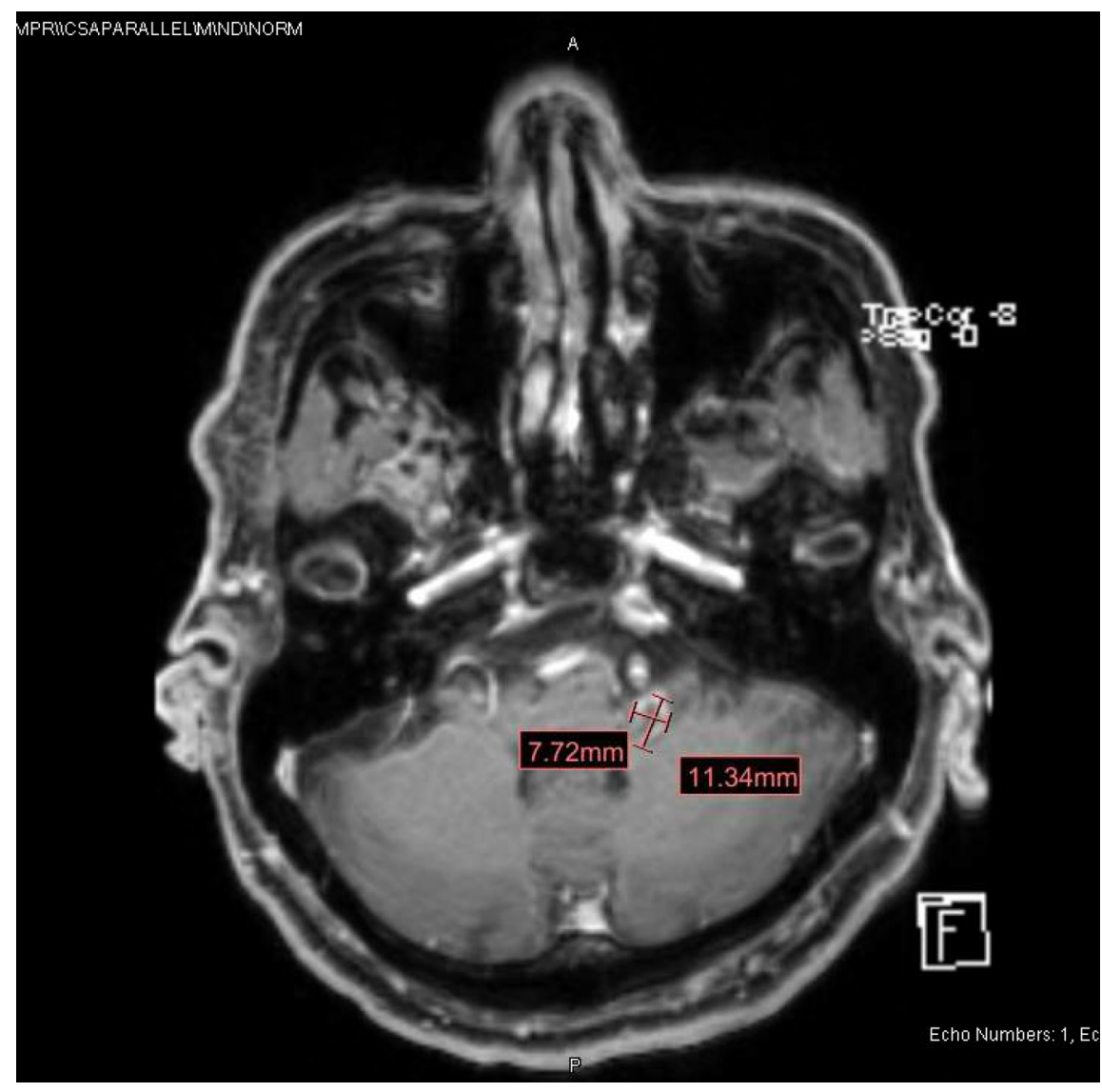

Figure 4: T1 weighted transverse MRI head showing cerebellar abscess (red cross).

\section{Discussion}

As we know from the basics of microbiology, Klebsiella pneumoniae is a gram-negative, facultative anaerobic, rod-shaped bacterium belonging to the Enterobacteriaceae genus. K. pneumoniae infections are usually hospital-acquired and occur in patients with impaired immunity. A unique exception to this is syndromes involving community-acquired primary liver abscesses which have been typically described in East Asian countries. ${ }^{1}$ We present a case of panophthalmitis and cerebellar abscess in association with a pyogenic liver abscess caused due to K. pneumoniae. Panophthalmitis differs from endophthalmitis due to inflammation extending to periocular tissue thereby involving all structures of the eyeball. A study on endogenous panophthalmitis described that 12 out of 18 patients with panophthalmitis had an infection caused by $K$. Pneumoniae, which was most commonly associated with liver abscesses. ${ }^{2}$ Our case adds to the list with an addition that the central nervous system (CNS) complications can occur concurrently along with panophthalmitis.

A Taiwanese retrospective cohort study of 177 cases of $K$. pneumoniae pyogenic liver abscess identified genotype $\mathrm{K} 1$ as a possible significant risk factor for causing ocular and CNS complications. ${ }^{1}$ Although the K1 serotype may be an important risk factor, the K2 serotype might also carry the potential of invasive disease. ${ }^{3}$ Cases of K2 serotype causing invasive liver abscess have been reported and it is certainly possible to develop metastatic infections in such instances. ${ }^{4,5}$ Diabetes is a notable risk factor for metastatic infectious disease as evident in our case. ${ }^{6,7}$ Genotype identification of Klebsiella was not done in this particular case, which is one of the limitations in this report. A 20-year retrospective study regarding K. pneumoniae endophthalmitis in association with pyogenic liver abscess concluded that only 5 patients out of 144 had more than one 
infectious focus; making it extremely rare. ${ }^{8}$ The same study also concluded that pyogenic liver abscessrelated endophthalmitis due to K. pneumoniae leads to poor visual outcomes. ${ }^{8}$ The right lobe of the liver is the most common site of abscess formation possibly due to typical unequal portal venous distribution from superior and inferior mesenteric vein. ${ }^{9}$

Management involves early initiation of intravenous antibiotics along with surgical or percutaneous drainage of the liver abscess to reduce morbidity and mortality. Prognosis in terms of visual outcome of K. Pneumoniae endophthalmitis is generally poor; prompt treatment is necessary. The patient may require evisceration or enucleation, but early vitrectomy may be useful in a select group of patients who do not respond to intravitreal antibiotics. $^{7}$

\section{Conclusion}

Endogenous panophthalmitis due to K. Pneumoniae is potentially fatal and monocular or binocular blinding complication of Pyogenic liver abscess. Diabetes and East Asian descent maybe a risk factor. Rarely multiple infection foci may be present as evident in our case with the presence of cerebellar abscess. Visual prognosis is generally guarded but a subset of patients with endophthalmitis may have preserved vision provided early medical and surgical intervention is done.

\section{Declarations :}

\section{Ethics approval and consent to participate}

Medical research committee at Hamad Medical Corporation approved the case study for publication. ( MRC-04-20-582)

\section{Consent for publication}

Written informed consent was obtained from the patient for publication of this case report. A copy of the written consent is available for review by the Editor-in-Chief of this journal.

\section{Availability of data and materials}

Data and materials regarding the case report are available to the Editor-in-Chief and can be requested from the corresponding author.

\section{Competing interests}

The authors have no conflict of interest relevant to this case.

\section{Funding}

Qatar National library funded the open access publication fees of this case.

\section{Author contributions}

The first authors (RS and SS) contributed equally to the writing and preparation of this article. RS and SS have written the initial draft of the manuscript and performed the literature review. The draft was revised and updated by RS and SS with supervision from WHI. RS, WR, ISK, IMA and WHI were part of the medical treating team. All the authors critically reviewed the initial and the final draft of the manuscript and approved it for submission.

\section{Appendix 1}

\begin{tabular}{lll}
\hline Name & Location & Contribution \\
\hline Rohit Sharma & Hamad Medical Corporation, Doha, Qatar & Writing the initial draft of the manuscript, M \\
Sundus Sardar & Hamad Medical Corporation, Doha, Qatar & Writing the initial draft of the manuscript, Re \\
Waail Rozi & Hamad Medical Corporation, Doha, Qatar & Medical management of the case, Revising the \\
Ibrahim Sa'id Khamees & Hamad Medical Corporation, Doha, Qatar & Medical management of the case, Revising the
\end{tabular}




\begin{tabular}{lll}
\hline Name & Location & Contribution \\
\hline Ibrahim Mohammad Obeidat & Hamad Medical Corporation, Doha, Qatar & Medical management of the case, Revising the \\
Wanis Hamad Ibrahim & Hamad Medical Corporation, Doha, Qatar & Conceptualization and supervision, Medical m \\
\hline
\end{tabular}

\section{Acknowledgements}

Not applicable

\section{References}

1. Fang C-T, Lai S-Y, Yi W-C, Hsueh P-R, Liu K-L, Chang S-C. Klebsiella pneumoniae Genotype K1: An Emerging Pathogen That Causes Septic Ocular or Central Nervous System Complications from Pyogenic Liver Abscess. Clin Infect Dis . 2007;45(3):284-293. doi:10.1086/519262

2. Chen K-J, Chen Y-P, Chao A-N, et al. Prevention of Evisceration or Enucleation in Endogenous Bacterial Panophthalmitis with No Light Perception and Scleral Abscess. PLOS ONE . 2017;12(1):e0169603. doi:10.1371/journal.pone.0169603

3. Virulence of Klebsiella pneumoniae Serotype K2 Should Not Be Underestimated in K. pneumoniae Liver Abscess | Clinical Infectious Diseases | Oxford Academic. Accessed July 12, 2020. https://academic.oup.com/cid/article/45/11/1530/335510

4. Pichler C, Büchsel M, Rossen JW, et al. First report of invasive liver abscess syndrome with endophthalmitis caused by a K2 serotype ST2398 hypervirulent Klebsiella pneumoniae in Germany, 2016. New Microbes New Infect . 2017;17:77-80. doi:10.1016/j.nmni.2017.02.006

5. Seo R, Kudo D, Gu Y, et al. Invasive liver abscess syndrome caused by Klebsiella pneumoniae with definite K2 serotyping in Japan: a case report. Surg Case Rep . 2016;2. doi:10.1186/s40792-016-0201-2

6. Han SH. Review of hepatic abscess from Klebsiella pneumoniae. An association with diabetes mellitus and septic endophthalmitis. West J Med . 1995;162(3):220-224.

7. Sheu S-J, Kung Y-H, Wu T-T, Chang F-P, Horng Y-H. RISK FACTORS FOR ENDOGENOUS ENDOPHTHALMITIS SECONDARY TO KLEBSIELLA PNEUMONIAE LIVER ABSCESS: 20-Year Experience in Southern Taiwan. RETINA . 2011;31(10):2026-2031. doi:10.1097/IAE.0b013e31820d3f9e

8. Chen Y-H, Li Y-H, Lin Y-J, et al. Prognostic Factors and Visual Outcomes of Pyogenic Liver AbscessRelated Endogenous Klebsiella pneumoniae Endophthalmitis: A 20-year retrospective review. Sci Rep . 2019;9. doi:10.1038/s41598-018-37643-y

9. Rahimian J, Wilson T, Oram V, Holzman RS. Pyogenic Liver Abscess: Recent Trends in Etiology and Mortality. Clin Infect Dis . 2004;39(11):1654-1659. doi:10.1086/425616 